\title{
A Correlation of Polarized Light Extinctions With Crystal Orientation in 70 Nickel-30 Copper Alloy
}

\author{
H. C. Vacher
}

\begin{abstract}
After etching 70 nickel-30 copper alloy to produce an optically anisotropic surface, the orientations of 12 crystals were determined by the twin-boundary method, and the positions of polarized light extinctions (obtained at normal incidence with crossed nicols) were measured. A comparison of the orientations with the positions of the extinctions showed that one was near the cubic pole farthest from the surface normal. Little or no extinction was obtained when a (100) or (111) plane was neariy parallel to the surface. Study of the results indicated that the optical anisotropy probably was caused by parallel furrows formed by the tendency of the etching reagent to develop etch pits whose facets were parallel to cubic planes.
\end{abstract}

\section{Introduction}

Previous work by D. H. Woodard [1] ${ }^{1}$ at the National Bureau of Standards showed that the surface of isotropic Monel, the 70 nickel-30 copper alloy, was optically anisotropic after the surface had been polished electrolytically and etched with "Monel contrast solution" [2]. It was noted that extinctions obtained at normal incidence, with crossed nicols, were not uniform over a grain in a specimen that had been deformed plastically as it was for a grain in an annealed specimen. This nonuniform extinction of individual grains was interpreted as indicating differences in orientation resulting from inhomogeneous strain.

The brilliant contrast of the grains and sharpness of the extinctions suggested that polarized light might be useful in determining the orientation of individual crystals. Accordingly, a study was undertaken in order to obtain quantitative data on the relation of extinctions to crystalline orientation.

\section{Procedures and Results}

Coarse-grained Monel was required so that individual crystals could be identified easily after several polishing and etching treatments. Suitable specimens were obtained by subjecting $1 / 2^{-}$by 8 -in. strips of commercial Monel sheet, 0.1 -in. thickness, to several strain-anneal cycles. A cycle consisted of straining in tension, 0.6 to 0.8 percent, followed by annealing at $1,150^{\circ} \mathrm{C}$ for $16 \mathrm{hrs}$.

In order to determine whether or not the optical anisotropy was reproducible, a specimen was photographed before and after repolishing and reetching, taking care to replace the specimen at the same angular setting with respect to the plane of vibration. The repolishing treatment consisted of removing the etched surface with fine alumina. Inspection of the photographs, figure 1, shows that the degrees of contrast between crystals were in general reproduced. This was confirmed by measurements of the four extinction positions with respect to a line on another specimen which had been adjusted so that the line coincided with the direction of vibration of the incident light. The amount of rotation re-

\footnotetext{
1 Figures in brackets indicate the literature references at the end of this paper
}

quired to obtain an extinction is designated as $\phi$ and will be referred to as such in the remainder of the paper. Results obtained after two polishing and etching treatments are listed in table 1. It can be seen that values for $\phi$ could be reproduced to $\pm 4^{\circ}$. The degree of reproducibility was found to produce differences in shading equivalent to that shown in figure 1.

TABLE 1. Reproducibility of extinction positions after successive polishing and etching treatments

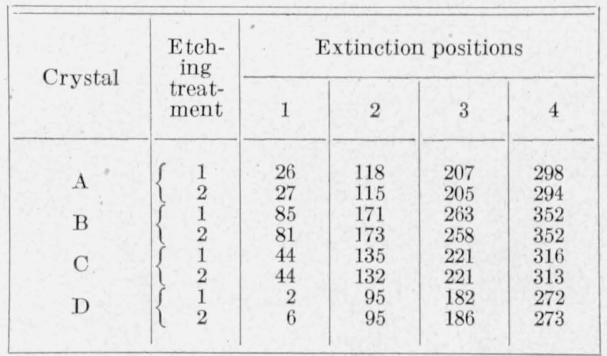

The Monel crystals were too small for their orientations to be determined conveniently by X-ray diffraction. Inspection of etched surfaces had shown many areas in which four first-order twins were present. Therefore, it was possible to determine the orientations of a cluster of crystals from the angles between their twin boundaries. By choosing crystal 1 , figure 2 , as the zero order, it followed that crystals $2,3,4$, and 5 were first-order twins, crystals $6,7,11$, and 12 were second-order twins and crystals 8,9 , and 10 were third-order twins. The angles between the reference boundary $1-3$ and the twin boundaries of crystals 1 to 12 are given in table 2 .

TABLE 2. Azimuth angles between twin boundaries of numbered crystals in figure 2

\begin{tabular}{|c|c|c|c|}
\hline Twin boundary & Azimuth a & Twin boundary & Azimuth a \\
\cline { 2 - 3 } & Degrees & & Degrees \\
& 0 & $4-6$ & 161 \\
$1-3$ & 110 & $4-7$ & 54 \\
$1-2$ & 66 & $7-8$ & 119 \\
$1-4$ & 48 & $7-9$ & 174 \\
$1-5$ & 71 & $7-10$ & 16 \\
$3-11$ & 115 & & \\
\hline
\end{tabular}

a Angles are measured clockwise from twin boundary 1-3, see figure 2 . 

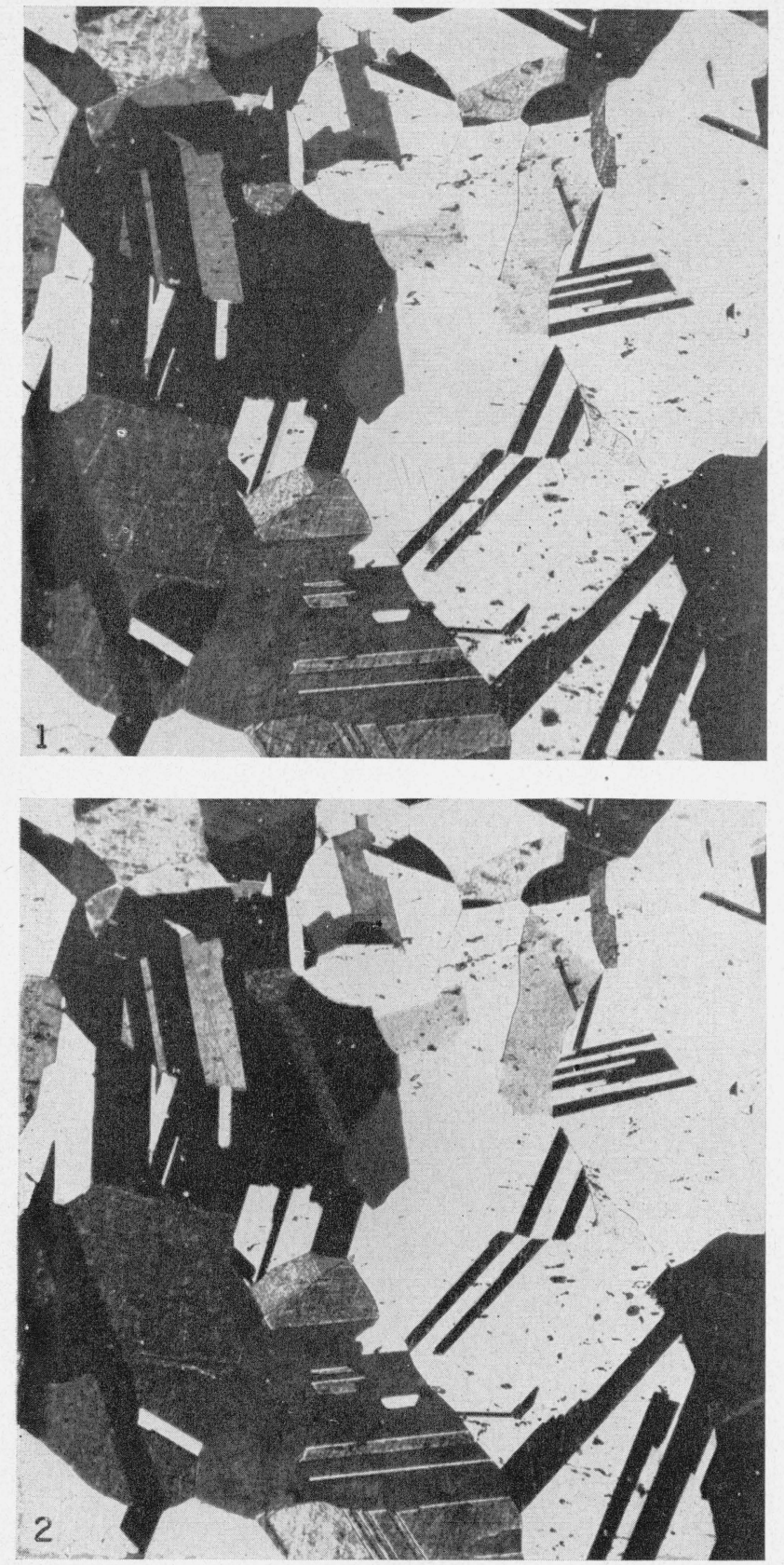

Figure 1. Reproducibility of intensity of reflections after repolishing and reetching.

Monel contrast solution, $\times 50$. 1 , Initial polishing and etching; 2 , after repolishng and reetching.

Taking advantage of the well known fact that in face-centered cubic metals, such as Monel, the twin boundaries are traces of (111) planes and using the procedure described by Barrett [3], the orientation of crystal 1 was determined, figure 3 . This procedure gave two solutions either of which would correspond to one of two orientations that would be obtained by plotting opposite poles on the same projection plane; however, the twin-trace method could be used be-

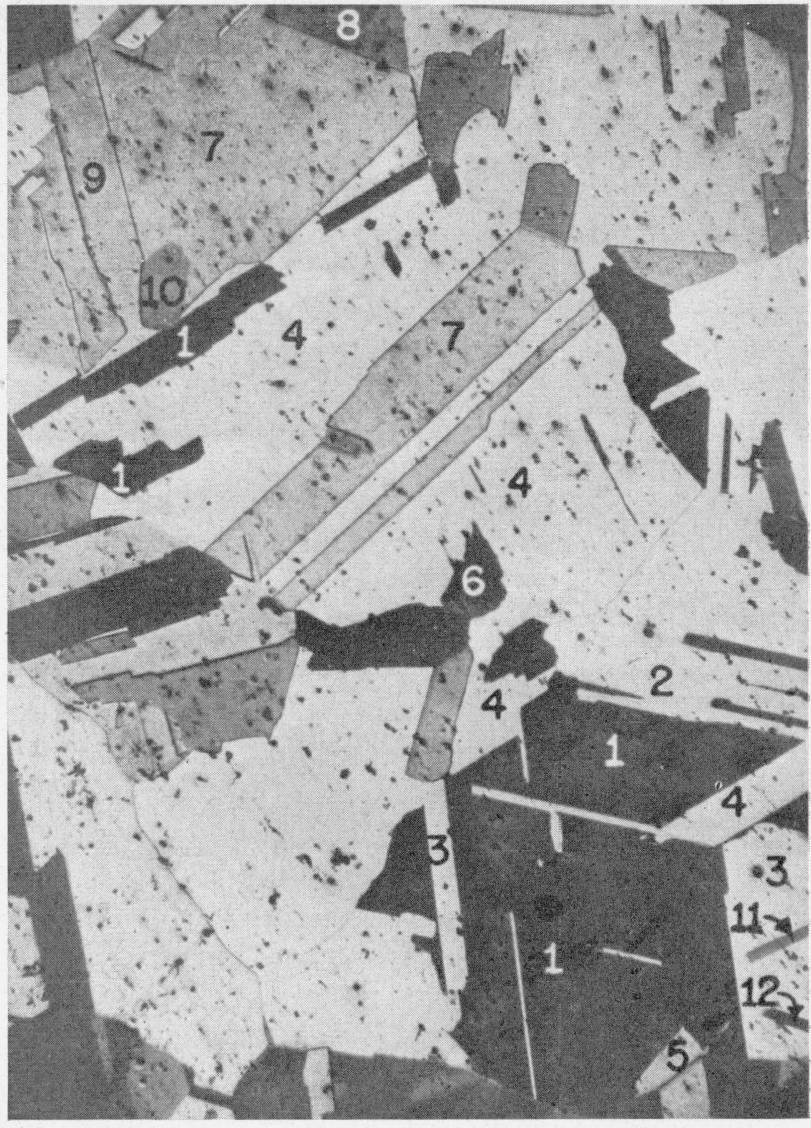

Figure 2. Crystals whose orientations were determined.

Monel contrast solution, $\times 50$.

cause opposite extinctions differed by $180^{\circ}$.

In order to verify the orientation of crystal 1 , the coincidence of the 111-poles of the first- and secondorder twins with the corresponding loci of normals of the observed twin boundaries was determined as indicated in figure 3 . The 111-poles of the first- and second-order twins were located with the aid of a standard stereographic projection, figure 4. This projection gives the angular relationships for the 100- and 111poles of a zero order crystal and its four first-order twins. A 100-pole of the zero order crystal coincides with the center of the projection. With this projection and a Wulff net, it was possible to locate the 100- and 111-poles of the first-order twins for any orientation of a zero-order crystal. By considering a first-order twin as a zero-order crystal, the 100and 111-poles of four second-order twins could be located. In this way 100- and 111-poles of highorder twinning could be located. The coincidence of the 111-poles with corresponding loci of normals to the observed twin boundaries was good, thereby checking the orientation assigned to crystal 1 .

After determination of the orientations of crystals 1 to 12 , their four extinction positions were measured. Average $\phi$ values differed by $90^{\circ}$ within the experimental error; therefore, they were adjusted so that the difference would be $90^{\circ}$. Crystals $1,2,3$, and 


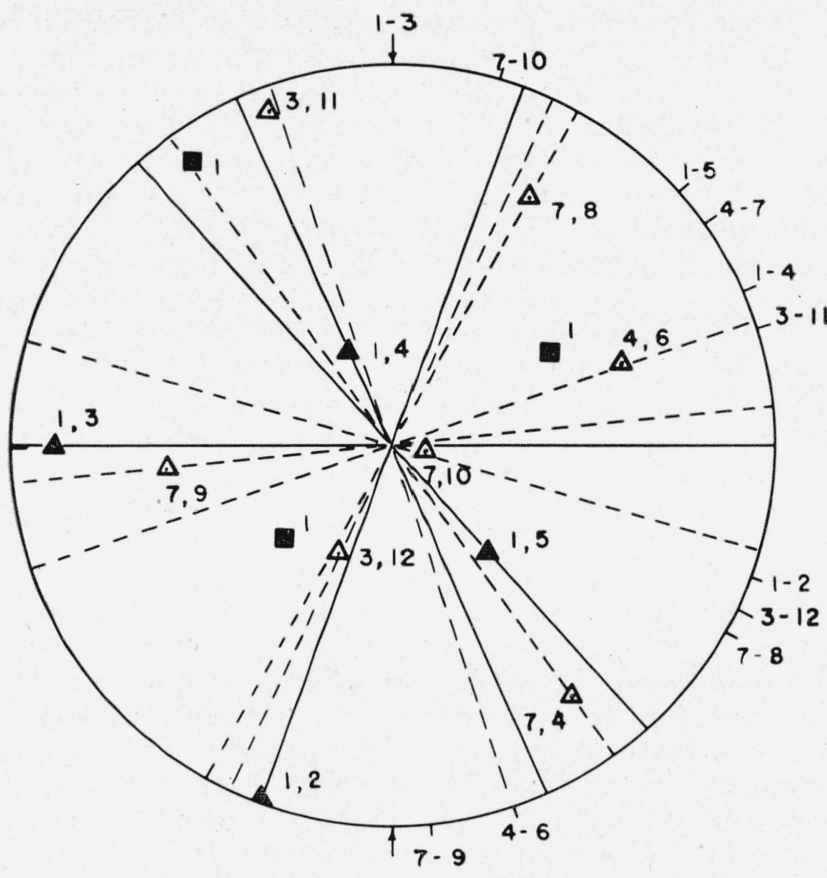

FiguRE 3. Determination of orientation by twin-boundary method.

(1) Angles between twin-boundaries are indicated at the periphery; (2), the solid meridians are loci of normals corresponding to the twin boundaries of erysta 1; (3), the solid triangles and squares indicate 111- and 100-poles, respectively, of crystal 1 as determined by its twin boundaries: (4), dashed meridians are loci of normals corresponding to the twin-bound aries of the first-and second-order twins: (5) the open triangles are the 111-poles of the first- and second-order twins of crystal 1, as determined by its orientation.

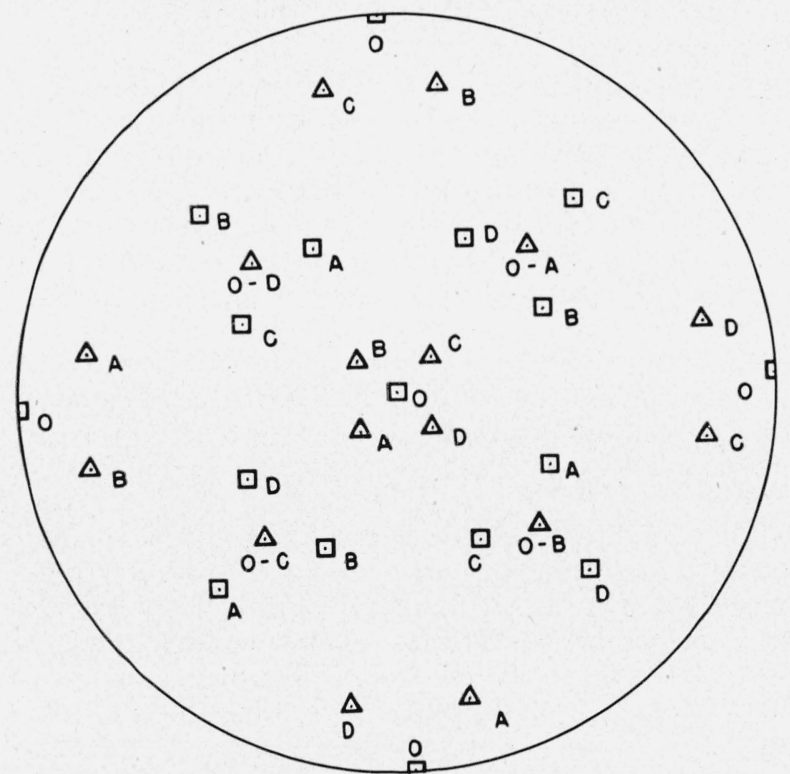

Figure 4. Standard stereographic chart of zero-and first-order twins.

Open triangles and squares are 111- and 100-poles, respectively. A, B, C, and $D$ identify first-order twins of crystal 0 .

11 gave sharp extinctions, therefore $\phi$ values were easier to obtain than for crystals $4,5,6,7,8$, and 12 , whose extinctions were less sharp. The values of $\phi$ for each crystal are listed in table 3 , together with the spherical coordinates of the cubic poles. The lati-

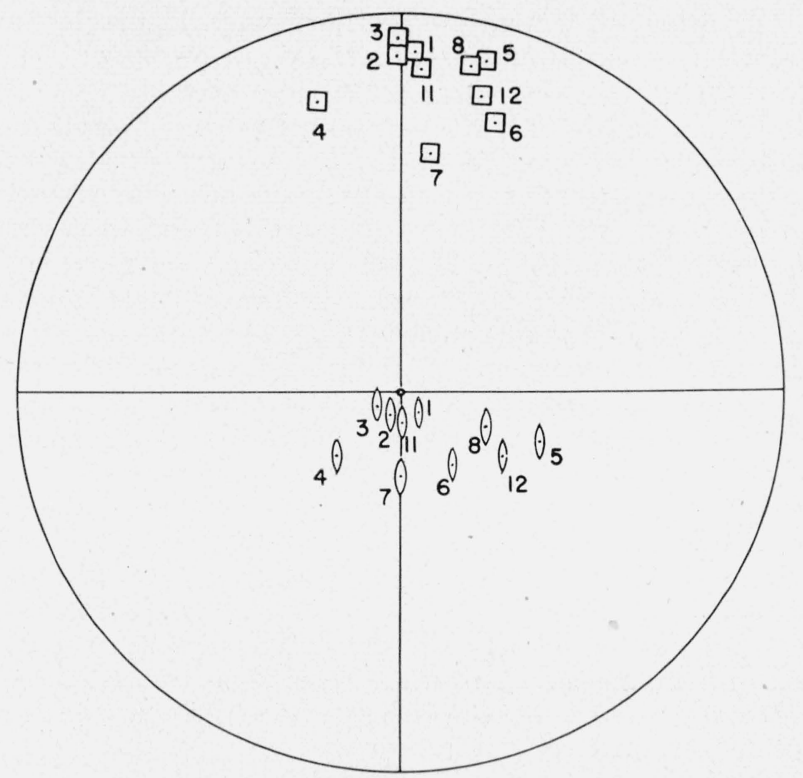

Figure 5. Correlation of crystal orientation and extinctions for numbered crystals in figure 2.

100-poles are indicated by squares, 110-poles by ellipses.

TABLE 3.-Extinctions and orientations of numbered crystals in figure 2

\begin{tabular}{|c|c|c|c|c|c|}
\hline \multirow{2}{*}{ Crystal } & \multirow{2}{*}{ Angles } & \multicolumn{4}{|c|}{ Extinetion positions } \\
\hline & & 1 & 2 & 3 & -4 \\
\hline \multirow{4}{*}{1} & $\phi$ & 53 & 143 & 233 & 323 \\
\hline & $\psi$ & 60 & -non & 227 & 325 \\
\hline & $\delta$ & 40 & & 50 & 6 \\
\hline & $\phi$ & 74 & 164 & 254 & 344 \\
\hline \multirow[t]{3}{*}{2} & $\psi$ & (n) & 157 & 253 & 351 \\
\hline & $\delta$ & & 41 & 7 & 48 \\
\hline & $\phi$ & 36 & 126 & 216 & 306 \\
\hline \multirow[t]{2}{*}{3} & $\psi$ & (n.... & 122 & 215 & 310 \\
\hline & $\delta$ & 30 & $\begin{array}{r}38 \\
120\end{array}$ & $\begin{array}{r}4 \\
210\end{array}$ & $\begin{array}{r}51 \\
300\end{array}$ \\
\hline \multirow[t]{3}{*}{4} & $\begin{array}{l}\phi \\
\psi\end{array}$ & $\begin{array}{l}30 \\
14\end{array}$ & 132 & & $\begin{array}{l}500 \\
278\end{array}$ \\
\hline & $\delta$ & 13 & 64 & & 22 \\
\hline & $\phi$ & 84 & 174 & 264 & 354 \\
\hline \multirow[t]{3}{*}{5} & $\psi$ & 98 & 189 & $\ldots+\ldots$ & 343 \\
\hline & $\delta$ & 6 & 13 & - n..... & 76 \\
\hline & $\phi$ & 20 & 110 & 200 & 290 \\
\hline \multirow[t]{2}{*}{6} & $\psi$ & 7 & 129 & 225 & (n) \\
\hline & $\delta$ & $\begin{array}{l}62 \\
80\end{array}$ & $\begin{array}{r}16 \\
170\end{array}$ & $\begin{array}{r}22 \\
260\end{array}$ & 350 \\
\hline \multirow[t]{3}{*}{7} & $\psi$ & 87 & $\begin{array}{l}170 \\
197\end{array}$ & 260 & $\begin{array}{l}350 \\
331\end{array}$ \\
\hline & $\delta$ & 25 & 37 & & 43 \\
\hline & $\phi$ & 58 & 148 & 238 & 328 \\
\hline \multirow[t]{2}{*}{8} & $\psi$ & 54 & 160 & 250 & (n....... \\
\hline & $\begin{array}{l}\delta \\
\phi\end{array}$ & $\begin{array}{l}71 \\
\text { (a) }\end{array}$ & (a) ${ }^{7}$ & $\begin{array}{l}18 \\
\text { (a) }\end{array}$ & (a) \\
\hline \multirow[t]{2}{*}{9} & $\begin{array}{l}\phi \\
\psi\end{array}$ & 40 & 130 & 223 & (a) \\
\hline & ${ }_{\phi}^{\delta}$ & $\begin{array}{r}6 \\
(\mathrm{a})\end{array}$ & (a) ${ }^{1}$ & $\begin{array}{l}84 \\
\text { (a) }\end{array}$ & \\
\hline \multirow[t]{3}{*}{10} & $\psi$ & 32 & 141 & 262 & \\
\hline & $\delta$ & 33 & 27 & 45 & \\
\hline & $\phi$ & 13 & 103 & 193 & 283 \\
\hline \multirow[t]{2}{*}{11} & $\psi$ & 23 & . & 186 & 286 \\
\hline & $\delta$ & 43 & 153 & $\begin{array}{r}45 \\
243\end{array}$ & $\begin{array}{r}9 \\
323\end{array}$ \\
\hline \multirow{2}{*}{12} & $\begin{array}{l}\phi \\
\psi\end{array}$ & 50 & $\begin{array}{l}105 \\
168\end{array}$ & $\begin{array}{l}243 \\
262\end{array}$ & 353 \\
\hline & $\delta$ & 66 & 12 & 21 & \\
\hline
\end{tabular}

2 Poor extinction.

tude, $\delta$, is referred to the polished surface and the azimuth, $\psi$, to the $1-3$ twin boundary. The small differences in brightness during a $360^{\circ}$ rotation for crystals 9 and 10 , made it impossible to obtain reproducible $\phi$ values. Therefore, no values are listed in table 3 . 
In order to determine if there was a correlation between extinctions and crystal orientation, the data for extinctions and orientations were plotted as shown in figure 5. For convenience in comparing differences between $\phi$ and $\psi$, the four extinctions were represented as mutually perpendicular meridians. Then for each crystal, the 100-pole making the smallest angle with the polished surface was plotted, using the nearest extinction as the fiducial line. The 110-pole nearest the surface normal also was plotted. Inspection of figure 5 shows that the 100 - and 110-poles of crystals 1, 2, 3, and 11, which have the sharpest extinctions, were near an extinction meridian and the surface normal, respectively. The results also show that if the 110-pole is displaced from the surface normal, then the reference extinction will be displaced to the opposite side of the 100-pole nearest the plane of the surface. These data are not sufficient to justify the statement of quantitative relationships, but it appears that there is a definite correlation between the orientation of a crystal and the location of the extinctions.

The suriace normal of crystal 9, which gave poor extinctions, was nearly parallel to a cubic plane. The crystallographic orientations of the surface normal for crystals 7 and 10 were nearly the same and near a 111-pole. However, the extinctions of crystal 10 were less sharp than those of crystal 7 . There was no apparent explanation for this inconsistency in the results. In general, the following could be stated: Sharp extinctions indicated that the surface was nearly parallel to a (110) plane. Very poor extinctions indicated that the polished surface was nearly parallel to either a (100) or a (111) plane.

\section{Discussion}

Recent reviews by Mott and Haines [4] and by Perryman [5] show that polarized light has been used in many investigations to reveal the polycrystalline nature of metals. In only a few cases $[6,7]$ was it demonstrated that polarized light could be used to obtain quantitative information on the orientation of crystals. The results in this paper show a correlation between extinctions and crystal orientation. Knowing the orientation, it is possible to locate the extinctions with a fair degree of accuracy and conversely a cubic pole can be located near one of the four extinctions. It is believed that further investigation will show that an empirical relationship can be worked out that will permit orientation of individual crystals in a polycrystalline metal to be determined more readily than is now possible, particularly by combining polarized light data with twin-boundary relationships or incomplete X-ray diffraction data.

In the earlier work [1] it was suggested that the optical anisotropy of the etched Monel surfaces was caused by an anisotropic film. Considering the work of Jones [8], Perryman and Lack [9], and the results described in this paper, it appears that the optical anisotropy can be explained equally well on the basis of the "ridged, or furrowed, structure" observed by Jones.
Jones observed striations on etched surfaces of certain metals that extinguished in polarized light at normal incidence when the striations were parallel or perpendicular to the vibration direction of the polarizer, the anlyzer being in the crossed position. In additional experiments with ordinary light at oblique incidence, Jones observed two bright reflections in a plane normal to the surface and to the striations. The normals to the reflecting planes differed by approximately $90^{\circ}$. Models were made from metal plates to simulate right-angle furrows. The models gave similar extinctions with crossed nicols. These observations led to the conclusion that the optical anisotropy was caused by a furrowed structure that was formed by the action of the etching reagents.

Perryman and Lack deposited a silver film, 800-A thickness, on a surface on Monel that had been prepared in a manner similar to that used in this work and found that the optical anisotropy was not destroyed. This experiment was repeated on an aluminum specimen, the surface of which had been anodized by a procedure developed by Hone and Pearson [10]. The same result was obtained. Similar films, deposited on suitably prepared surfaces on anisotropic cadmium and zinc specimens obliterated the optical anisotropy. They concluded from these results that the optical anisotropy of the etched Monel and anodized aluminum suriaces was caused by the shape of the surface contours and not by the anisotropy of a surface film. Hone and Pearson had shown that the anodized aluminum surface was striated but had concluded that the film was anisotropic.

The results summarized in figure 5 show close agreement between extinctions and meridians that can be passed through the surface normal and cubic poles for orientations approximating the "110-surface". 2 This also indicates that the anisotropy was caused by a "ridged or furrowed structure", as described by Jones [9], the sides of the furrows being parallel to cubic planes whose intersections were parallel to the surface. This structure satisfies the geometry necessary for a reflection from two surfaces to be coincident with the incident beam. However, if etch pits whose facets were parallel to cubic planes were perfect, the structure described by Jones could give a coincident double reflection only for orientations in which the surface normal was in a cubic plane. Figure 5 shows that this condition need not be satisfied in order to obtain sharp extinctions. In absence of data to the contrary, the optical anisotropy of crystals $4,5,6,7$, and 12 could be explained as being due in part to imperfection of the etch pits and in part to the fact that the incident beam was convergent. There is also the possibility of a striated film, such as the anodized aluminum surface, the directions of the striations being controlled by the general shape of the etch pits. As yet no direct evidence has been obtained to verify the presence of parallel furrows on etched Monel surfaces.

2 The crystallographic orientation of the surface normal is denoted by giving the indices of the plane that is parallel to the surface, immediately before the word "surface." 
When the etched surface is parallel to a (100) plane, only coincident single reflection is possible, and if plane polarized light is used, the reflected beam would be extinguished by the analyzer. This fact was used by Dunsmuir [7] to estimate the number of crystals in silicon iron sheet that approximated the 100-surface, assuming that all other orientations would give extinctions. This assumption apparently is not true, as the foregoing results showed that orientations approximating the 111surface also do not always show extinctions. The result of the following experiment also supports this conclusion. Impressions of a cube corner were made in a polished surface on a stainless steel specimen, keeping the cube diagonal normal to the surface during the compression. These impressions simulate etch pits having facets parallel to cubic planes in crystals having a 111-surface. The impressions did not show extinctions.

It appears probable then that the optical anisotropy of the Monel crystals was caused by imperfect etch pits that combined in such a way that parallel furrows were formed. On this basis, extinctions should be obtained when the furrows were parallel or perpendicular to the vibration direction of the polarizer, as is the case with parallel scratches.

\section{Conclusions}

The twin-boundary method provides a useful method for determining the orientation of crystals in annealed Monel.

The results indicate a qualitative relationship between crystal orientation and the extinctions of polarized light reflected from the crystals.

The optical anisotropy of etched Monel metal surfaces probably was caused by parallel furrows formed by the tendency of the etching reagent to develop etch pits whose facets were parallel to cubic planes.

D. H. Woodard, now a member of the staff of the Metallurgical Project, Massachusetts Institute of Technology, Cambridge, Mass., cooperated in some of the preliminary stages of this work.

\section{References}

[1] D. H. Woodard, Stages in the deformation of Monel metal as shown by polarized light, Am. Inst. Min. Met. Engrs., Metals Trans. 185, 722 (1949).

[2] David I. Sinizer, The metallography of nickel and nickel alloys, Metals Handbook, 1948 Ed., p. 1048 (Am. Soc. Metals, Cleveland, Ohio).

[3] C. S. Barrett, The structure of metals, p. 40 (McGrawHill Book Co., New York, N. Y., 1943).

[4] B. W. Mott and R. H. Haines, Examination of metals under polarized light. I. Theory and apparatus, Research 4, 24 (Jan. 1951) ; II. Applications, Research 4, 63 (Feb, 1951)

[5] E. C. W. Perryman, Recent development in metallography, Metal Ind. 79, 71 (July 27, 1951).

[6] P. F. George, Some special metallographic techniques for magnesium alloys, Trans. Am. Soc. Metals 38, 687 (1947).

[7] P. G. Dunsmuir, A summary of the proceedings of the conference on the examination of metals by optical methods, British Iron and Steel Research Association Report, p. 51 (May 1949).

[8] Olwen Jones, Reflection of plane polarized light by etched metals, Phil. Mag. 48, 207 (1924)

[9] E. C. W. Perryman and J. M. Lack, Examination of metals by polarized light, Nature 167, 479 (March 24, 1951).

[10] A. Hone and E. C. Pearson, Grain orientation in aluminum revealed by anodic film, Metal Progress 53, 363 (March 1948).

Washington, July 1, 1952. 